\title{
Who are at high risk of mortality and morbidity among children with congenital heart disease undergoing noncardiac surgery?
}

\author{
In-Kyung Song and Won-Jung Shin
}

Received November 25, 2020

Department of Anesthesiology and Pain Medicine, Laboratory for Cardiovascular Dynamics, Asan Medical Center, University of Ulsan College of Medicine, Seoul, Accepted December 7, 2020 Korea

\section{Corresponding author \\ Won-Jung Shin, M.D., Ph.D. Department of Anesthesiology and Pain Medicine, Laboratory for Cardiovascular Dynamics, Asan Medical Center, University of Ulsan College of Medicine, 88 Olympic-ro 43-gil, Songpa-gu, Seoul 05505, Korea}

Tel: 82-2-3010-5644

Fax: 82-2-3010-6790

E-mail: wjshin@amc.seoul.kr
With advances in the development of surgical and medical treatments for congenital heart disease (CHD), the population of children and adults with $\mathrm{CHD}$ is growing. This population requires multiple surgical and diagnostic imaging procedures. Therefore, general anesthesia is inevitable. In many studies, it has been reported that children with CHD have increased anesthesia risks when undergoing noncardiac surgeries compared to children without CHD. The highest risk group included patients with functional single ventricle, suprasystemic pulmonary hypertension, left ventricular outflow obstruction, and cardiomyopathy. In this review, we provide an overview of perioperative risks in children with CHD undergoing noncardiac surgeries and anesthetic considerations in patients classified as having the highest risk.

Keywords: Anesthesia; Child; Congenital heart defect; Risk.

\section{INTRODUCTION}

The incidence of congenital heart disease (CHD) is reported to be about 6 per 1,000 full-term live births in the United States [1]. With advances in the perinatal diagnosis of CHD and improvement in surgical and medical management, the survival rate and life expectancy in children with CHD have been increasing [2]. These children frequently require noncardiac surgeries, including laparoscopic, urogenital, and otolaryngological surgeries. During the first year of life, $41 \%$ of infants who underwent congenital heart surgery had undergone noncardiac surgery by the age of 5 years [3]. With an increasing demand for surgical procedures under general anesthesia in these patients, it is not uncommon for anesthesiologists to encounter children with an unrepaired CHD or residual pathologic conditions, as well as children with a repaired CHD. Therefore, it is important to identify children at risk of perioperative morbidity and mortality and to understand their pathophysiologic and hemodynamic status when preparing their general anesthetic plan. In the following review, we discuss the current knowledge regarding children with CHD who have high anesthesia and surgical risks and also focus on the perioperative considerations for these high-risk patients.

\section{RISKS OF NONCARDIAC SURGERIES IN CHILDREN WITH CHD}

Recently, mortality and perioperative adverse events related to noncardiac surgeries have been reported in children studies with large sample populations, including those with and without CHD. Baum et al. [4] showed that the overall 30-day mortality after noncardiac procedures was higher in patients with CHD (6.0\%) than in those without CHD (3.8\%). They also found that age, complexity of

This is an Open Access article distributed under the terms of the Creative Commons Attribution Non-Commercial License (http://creativecommons.org/licenses/by-nc/4.0) which permits unrestricted non-commercial use, distribution, and reproduction in any medium, provided the original work is properly cited.

Copyright (c) the Korean Society of Anesthesiologists, 2021 
the operation, and CHD, were associated with perioperative mortality. In the Pediatric Perioperative Cardiac Arrest (POCA) Registry, causes of anesthesia-related cardiac arrest were reported from nearly 80 voluntarily enrolled North American institutions that provide anesthesia for children over a period of 10 years [5]. In this study, children with CHD had a higher mortality rate (33\%) than those without CHD (26\%). Cardiac arrest was strongly associated with surgical complexity and the patient's underlying functional status. Among the types of CHD, single ventricular physiology, aortic stenosis, and cardiomyopathy were associated with the highest mortality following a cardiac arrest. In another study, the incidence of perioperative cardiac arrest was 2.9 per 10,000 anesthetic episodes among patients undergoing noncardiac surgeries, and of these, $27 \%$ had CHD [6]. In addition, they found that the most common causes of cardiac arrest during noncardiac surgeries were hypovolemia, bleeding, and massive transfusion. These findings suggest that intrinsic surgical factors and the associated hemodynamic deterioration are important for estimating the risk of cardiac arrest. In a study investigating 101,885 anesthetic cases, the overall 24 -h mortality after anesthesia was 13.4 per 10,000 [7]. The highest incidence of death was in children younger than 30 days. Collectively, children with CHD are faced with a high risk of mortality and adverse events related to anesthesia when undergoing noncardiac surgeries. In particular, the complexity of CHD is regarded as an important factor influencing high risk of mortality. Therefore, children with CHD may be at higher risk of mortality and morbidity during and after noncardiac surgeries. In addition, age, anatomical and functional status according to CHD complexity, and intrinsic surgical risks are points that must be considered with care when estimating the risks of mortality and morbidity.

The other issue to consider is how to define and stratify the risk factors. According to the American College of Surgeons who collected data on noncardiac surgeries as a part of its National Surgical Quality Improvement Program for the classification of CHD, CHD can be stratified as minor, major, and severe, based on the residual lesion burden and the functional status of the heart [8]. According to this classification, children who have maintained good cardiac conditions with or without medication and children with a repaired CHD are classified as having minor CHD; patients with a repaired CHD but who have residual abnormalities in hemodynamic status are considered to have major CHD; patients with an unrepaired cyanotic CHD, pulmonary hypertension, or ventricular dysfunction or children awaiting transplantation are classified to have severe CHD. After propensity matching for age, sex, physical status, surgical emergency, and surgical complexity, severe CHD was significantly associated with 30 -day mortality and overall mortality [8]. However, there was no difference between children with minor CHD and their matched controls. In addition to perioperative mortality, morbidities including postoperative reintubation, infections, renal failure, neurologic complications, thrombotic events, reoperation, and readmission were more frequent in patients with major and severe CHD. In a recent study regarding surgical complexity, children with CHD younger than 1 year showed a greater risk of postoperative complications, with an incremental increase in odds ratios in the order of minor, major, and severe CHD [9]. In another study of 3,010 children with CHD undergoing noncardiac surgeries, major and severe CHD remained significant risk factors for perioperative cardiovascular events after adjusting for the American Society of Anesthesiologists physical status, emergency cases, and surgical types [10].

In a previous study using a risk stratification tool to classify risk levels for perioperative cardiac complications, repaired atrial defects and ventricular septal defects were considered low risk, maintenance cardiac medications; and repaired cyanotic or complex CHD were classified as moderate risk; and unrepaired cardiac anomalies, Williams syndrome, pulmonary hypertension, valvular heart disease with significant valvular gradients, hypertrophic cardiomyopathy with obstruction, congestive heart failure, or children with ventricular-assisted devices were considered high risk [11]. To further determine the anesthesia risk, age less than 1 year, comorbidities, and surgical complexity were included as the next step. Similarly, results from non-validated data of anesthesia for noncardiac surgeries indicated that children with CHD were classified as low, intermediate, and high risk, and further discriminated based on physiological decompensation, complexity of the CHD, major surgery, age under 2 years, emergency, preoperative hospital stay more than 10 days, and American Society of Anesthesiologists physical status [12,13].

To date, only one study has reported a risk assessment model using a validation cohort. This study identified eight preoperative factors that were significant in determining in-hospital mortality: 1) emergency procedure, 2) severe $\mathrm{CHD}, 3)$ previous surgery within the last 30 days, 4) single 
ventricular physiology, 5) inotropic use, 6) cardiopulmonary resuscitation, 7) kidney injury, and 8) mechanical ventilation [14]. Based on the variables obtained from multivariable logistic regression analyses, scores from 0 to 10 were determined. This scoring system showed good discrimination and calibration with an area under the receiver operating characteristic curve of 0.831 (95\% confidence interval: $0.787-0.875)$ in the validation cohort. Briefly, scores $\leq 3$ were associated with low risk, scores of 4-6 were associated with medium risk, and scores $\geq 7$ were associated with a high risk for mortality (odds ratios 1.54, 4.19, and 22.15, respectively) [14]. Notably, major and severe CHD, including single ventricular physiology, were found to be major determinants of perioperative outcomes [14]. In contrast, surgical complexity was not significant [14]. They also highlighted that scoring-based risk stratification for mortality may be necessary to help guide the perioperative management of patients with high-risk CHD.

Herein, we reviewed the perioperative considerations of children undergoing noncardiac surgeries who were classified as having high-risk CHD, in common with most of the previously reported studies.

\section{SINGLE VENTRICULAR PHYSIOLOGY}

It is critical that anesthesiologists understand the physiology of each palliative stage of a single ventricle, which includes truncus arteriosus, large and multiple ventricular septal defects, and hypoplastic left heart syndrome (HLHS). Patients who have not undergone completion of superior cavopulmonary anastomosis (SCPA) are known to have the highest risks during noncardiac surgeries and congenital heart surgery. Especially in HLHS, the mortality rate of patients younger than 2 years has been reported to be up to $19 \%$ after noncardiac surgeries [15]. Hemodynamic derangement is caused by excessive pulmonary blood perfusion and poor systemic perfusion from imbalanced circulation, decreased coronary perfusion, impairment of the systemic right ventricle, and atrioventricular valve dysfunction. As the single ventricle concurrently operates both the pulmonary and systemic circulations, hemodynamic balance is frequently disrupted by alterations in pulmonary and systemic vascular resistance (PVR and SVR, respectively), ventilatory strategy including hypoxia and hypercapnia, acid-base balance, and intravascular volume status. Therefore, postponing elective noncardiac procedures under after SCPA is recommended. If the patient could not postpone due to an emergent condition, Pulmonary-to-systemic blood flow ratio should be at or just below 1 to maintain systemic perfusion and optimize oxygen delivery, consequently resulting in arterial oxygen saturation of $80-90 \%$ [16].

After the SCPA, the single ventricle is no longer operating with the volume overloading required to sustain parallel circulations. Accordingly, cardiac output and systemic perfusion are not entirely dependent on pulmonary blood flow, which makes the hemodynamic performance relatively stable. However, hypercarbia, acidosis, and elevated airway pressure should be avoided because pulmonary blood flow remains dependent on PVR. However, hypocarbia may exacerbate a decrease in cerebral blood flow and reduce venous return from the brain and upper body [17]. If there is high pressure in the superior vena cava, the head and the tongue may become congestive due to disturbance in venous return. In these patients, the goal of anesthetic management is to maintain systemic oxygenation with adequate pulmonary blood flow, which is secured by optimizing intravascular volume, minimizing airway pressure, and ensuring a low PVR.

Finally, the destination of single ventricular physiology is the completion of successful Fontan circulation, in which all systemic venous return is composed of pulmonary blood flow. Even though systemic arterial saturation is maintained above $90 \%$, cardiac output relies on pulmonary blood flow that runs down passively because there is no pump function producing a pulsatile driving pressure. Pulmonary blood flow and cardiac output are strictly influenced by mechanical ventilation with positive end-expiratory pressure $[18,19]$. In addition, the pressure of the systemic venous system is elevated, and its capacitance is decreased, and accompanied by diminished recruitment reserve of the vascular volume [20]. Consequently, patients with a Fontan circulation are intolerant of vasodilation from anesthetic agents, surgical bleeding, and dehydration. It may be beneficial to prepare inotropic agents and vasopressors for the treatment of hypotension and avoid excessive volume challenges [20]. Unfortunately, bleeding tendency may be increased due to persistent high venous pressure and anticoagulant therapy. However, they also have thrombosis risks [21]. Along with preloading, other factors are required to achieve the perfect Fontan circulation as follows: low PVR, sinus rhythm, normal atrioventricular valve function, good ventricular performance, and the absence of inflow and outflow tract obstruction [22] (Fig. 1). After surgery, it 


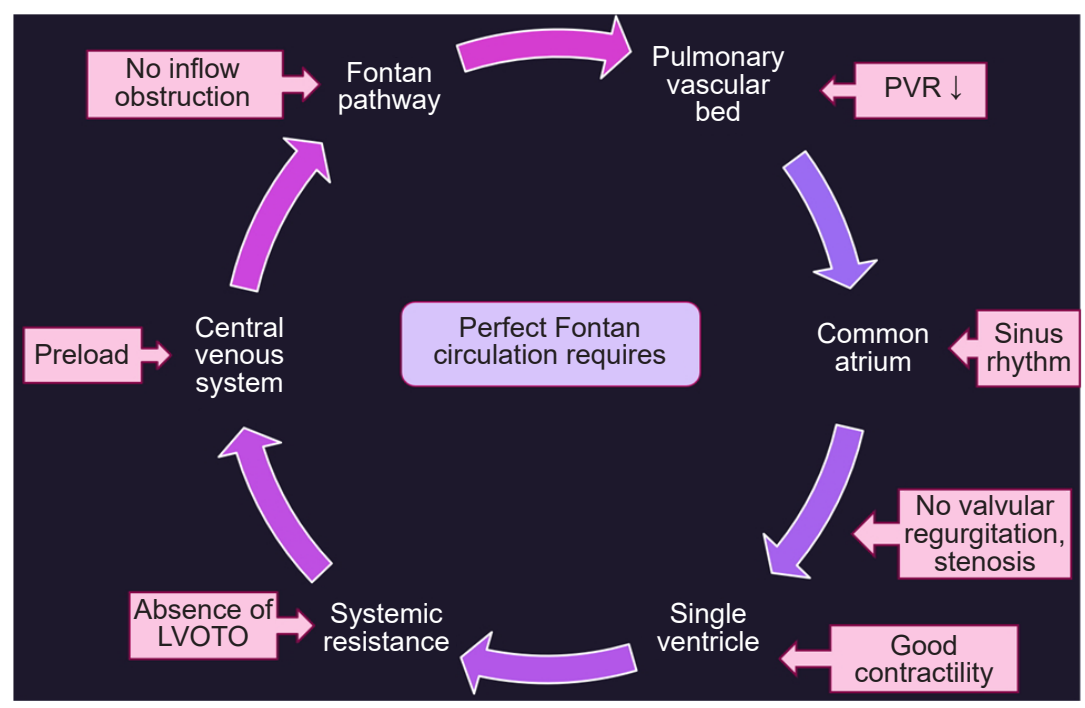

Fig. 1. Requirements for a perfect Fontan circulation. Factors at each anatomical structure are essential to secure successful Fontan circulation: an adequate preload, low pulmonary vascular resistance (PVR), normal sinus rhythm, normal atrioventricular valve function, good ventricular contractility, and absence of inflow and left outflow tract obstruction (LVOTO).

can be favorable for patients with Fontan physiology to extubate as early as possible and restore spontaneous breathing.

\section{SUPRASYSTEMIC PULMONARY HYPERTENSION}

Pulmonary hypertension is one of the factors associated with a high perioperative mortality rate. The perioperative mortality risk has been reported to be at least 20 -fold greater in children with pulmonary hypertension than in those without among children undergoing noncardiac procedures [23]. Suprasystemic pulmonary hypertension is defined as the ratio of mean or systolic pulmonary artery pressure to systemic artery pressure (PAP/SAP) of $>100 \%$. As pulmonary hypertension progresses, perioperative outcomes worsen. Thus, it is crucial to determine the severity of pulmonary hypertension during preoperative evaluation. Features that distinguish the severity of pulmonary hypertension are as follows: right ventricular (RV) dysfunction on echocardiography, decreased functional capacity, growth failure, significantly elevated brain natriuretic peptide (BNP) or N-terminal pro-BNP levels, and poor catheterization indices [24]. In these children who are chronically exposed to suprasystemic PAP, acute RV failure and pulmonary hypertensive crisis may occur given the limited functional reserve of the RV. If coronary hypoperfusion occurs simultaneously, catastrophic results such as myocardial ischemia, fatal arrhythmia, and cardiac arrest may oc- cur even if the increase in PAP is small [25].

Whenever patients undergo surgery and general anesthesia, pulmonary hypertensive crisis can develop due to various causes including hypoxia, hypercarbia, acidosis, hypothermia, and sympathetic stimulation. Particularly in children whose PVR increases but remains responsive and modifiable, any causes that induce an increase in PVR can trigger a vicious cycle of pulmonary hypertensive crisis [25] (Fig. 2). During anesthesia, clinical signs manifest as arterial desaturation and low end-tidal $\mathrm{CO}_{2}$ levels due to impaired pulmonary blood flow, sudden cardiovascular collapse, hypotension, and tachy- or brady-arrhythmia. Pulmonary hypertensive crisis should be treated promptly. Management of pulmonary hypertensive crisis may involve ventilation with $100 \%$ inspired $\mathrm{O}_{2}$, mild hyperventilation, inhaled nitric oxide, alkalinization using sodium bicarbonate infusion, and inotropic support.

\section{LEFT VENTRICULAR OUTFLOW TRACT OBSTRUCTION}

According to the POCA registry, $16 \%$ of anesthesia-related cardiac arrest was caused by obstruction to ventricular outflow such as supravalvular, subaortic, or aortic stenosis [5]. After cardiac arrest in these patients, the mortality rate was $62 \%$, suggesting that anesthesiologists should be meticulous in perioperative management. In patients with Williams syndrome, cardiovascular abnormalities are characterized by supravalvular aortic and pulmonary stenoses 


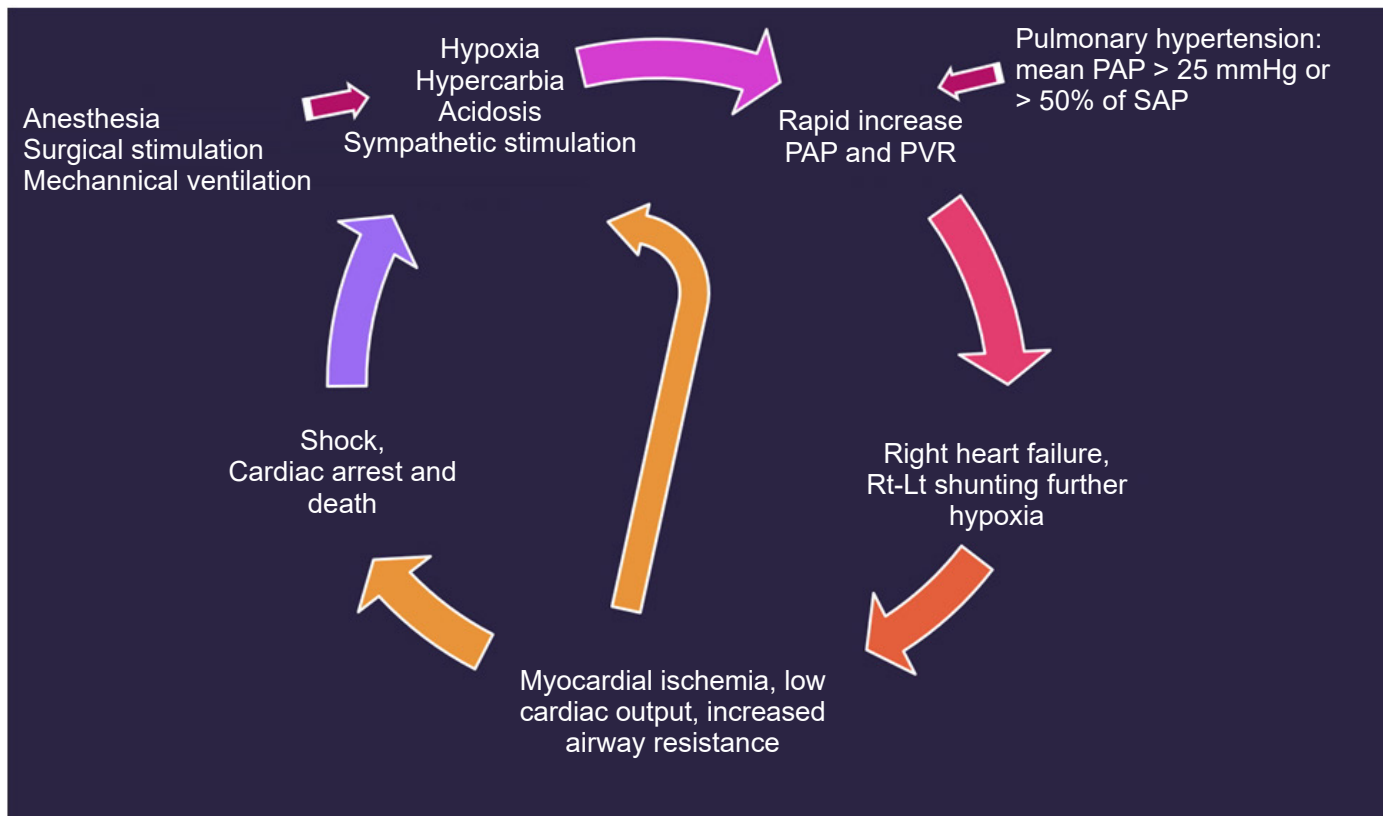

Fig. 2. A vicious cycle of pulmonary hypertensive crisis. During general anesthesia and surgical procedures, conditions of hypoxia, hypercarbia, acidosis, hypothermia, and sympathetic stimulation can induce a further increase in pulmonary artery pressure (PAP) and pulmonary vascular resistance (PVR), thereby triggering a vicious cycle of pulmonary hypertensive crisis. SAP: systemic artery pressure.

with elastin arteriopathy. Ventricular outflow obstruction is followed by myocardial hypertrophy. Worsening biventricular hypertrophy may lead to sudden cardiovascular collapse in patients undergoing anesthesia. Moreover, factors related to coronary blood flow may also contribute to cardiovascular events during the perioperative period including 1) Anatomical abnormalities and coronary artery stenosis, 2) compromise of diastolic blood pressure caused by loss of aortic distensibility, and 3) myocardial oxygen imbalance from increased demand of hypertrophied myocardium [26]. Prolongation of the corrected QT interval is present in $13 \%$ of patients with Williams syndrome, and this is associated with sudden cardiac arrests [27]. According to examinations conducted as a part of preoperative evaluation, children with Williams syndrome can be classified to have a low, moderate, or high risk. However, regardless of the risk classification, anesthetic management is performed while attempting to maintain sinus rhythm, and ensuring the maintenance of contractility, restoration of intravascular volume deficit, and preservation of SVR [27]. Therefore, the choice of anesthetic agents must be guided by whether a drug induces abrupt hemodynamic perturbation.

\section{CARDIOMYOPATHY}

Children with cardiomyopathy and ventricular dysfunc- tion are classified to have high perioperative mortality and morbidity risks related to anesthesia [8]. Based on the POCA registry, cardiomyopathy contributed to $13 \%$ of perioperative cardiac arrests [5]. The etiology of cardiomyopathy includes idiopathic causes (hypertrophic, restrictive, and dilated), structural heart disease (such as CHD including single ventricular physiology), and secondary disorders (such as end-stage renal disease and congenital heart block) [28]. Among these children, there may be an increased risk of preoperative morbidity and mortality when ventricular dysfunction is caused by dilated cardiomyopathy, failing Fontan circulation, left ventricular outflow obstruction, and pulmonary hypertension. General anesthesia may induce hemodynamic instability even at regular doses of anesthetic agents because ventricular functional reserve is severely compromised. Ketamine is recommended as the choice of induction agent because the sympathetic tone is preserved. In addition, balanced anesthesia is beneficial for achieving hemodynamic stability using opioids, volatile agents, neuromuscular blockade, or a combination of these agents [29]. In children with cardiomyopathy, the anesthetic goal is to maintain the preload, sinus rhythm, SVR, ventricular contractility, and coronary perfusion. Inotropic and vasoactive drugs may be frequently required to manage hypotension and low cardiac output. It is important that an excessively elevated SVR be avoided because an impaired ventricle with limited 
contractile reserve is not tolerant of a high afterload [30].

\section{CONCLUSION}

Children with CHD, particularly single ventricular physiology, suprasystemic pulmonary hypertension, left ventricular outflow obstruction, and cardiomyopathy with ventricular dysfunction, have the highest morbidity and mortality risks following noncardiac surgeries. During the preoperative evaluation of these patients, it is necessary to identify whether residual functional or anatomical impairment is present at the time of surgery. To prevent poor outcomes and avoid worse-case scenarios, anesthesiologists should be fully acquainted with the pathophysiology of CHD and be able to respond to intraoperative events and complications during surgery in a timely manner.

\section{CONFLICTS OF INTEREST}

No potential conflict of interest relevant to this article was reported.

\section{AUTHOR CONTRIBUTIONS}

Conceptualization: Won-Jung Shin, In-Kyung Song. Writing - original draft: In-Kyung Song. Writing - review \& editing: Won-Jung Shin.

\section{ORCID}

In-Kyung Song, https://orcid.org/0000-0002-7699-2005 Won-Jung Shin, https://orcid.org/0000-0002-6790-3859

\section{REFERENCES}

1. Hoffman JI, Kaplan S. The incidence of congenital heart disease. J Am Coll Cardiol 2002; 39: 1890-900.

2. Marelli AJ, Ionescu-Ittu R, Mackie AS, Guo L, Dendukuri N, Kaouache M. Lifetime prevalence of congenital heart disease in the general population from 2000 to 2010. Circulation 2014; 130: 749-56.

3. Sulkowski JP, Cooper JN, McConnell PI, Pasquali SK, Shah SS, Minneci PC, et al. Variability in noncardiac surgical procedures in children with congenital heart disease. J Pediatr Surg 2014; 49: $1564-9$

4. Baum VC, Barton DM, Gutgesell HP. Influence of congenital heart disease on mortality after noncardiac surgery in hospi- talized children. Pediatrics 2000; 105: 332-5.

5. Ramamoorthy C, Haberkern CM, Bhananker SM, Domino KB, Posner KL, Campos JS, et al. Anesthesia-related cardiac arrest in children with heart disease: data from the Pediatric Perioperative Cardiac Arrest (POCA) registry. Anesth Analg 2010; 110: 1376-82.

6. Flick RP, Sprung J, Harrison TE, Gleich SJ, Schroeder DR, Hanson AC, et al. Perioperative cardiac arrests in children between 1988 and 2005 at a tertiary referral center: a study of 92,881 patients. Anesthesiology 2007; 106: 226-37.

7. van der Griend BF, Lister NA, McKenzie IM, Martin N, Ragg PG, Sheppard SJ, et al. Postoperative mortality in children after 101,885 anesthetics at a tertiary pediatric hospital. Anesth Analg 2011; 112: 1440-7.

8. Faraoni D, Zurakowski D, Vo D, Goobie SM, Yuki K, Brown ML, et al. Post-operative outcomes in children with and without congenital heart disease undergoing noncardiac surgery. J Am Coll Cardiol 2016; 67: 793-801.

9. Miller R, Tumin D, Tobias JD, McKee C. Estimating surgical risk in younger and older children with congenital heart disease. J Surg Res 2018; 232: 298-307.

10. Lee S, Reddington E, Koutsogiannaki S, Hernandez MR, Odegard KC, DiNardo JA, et al. Incidence and risk factors for perioperative cardiovascular and respiratory adverse events in pediatric patients with congenital heart disease undergoing noncardiac procedures. Anesth Analg 2018; 127: 724-9.

11. Saettele AK, Christensen JL, Chilson KL, Murray DJ. Children with heart disease: risk stratification for non-cardiac surgery. J Clin Anesth 2016; 35: 479-84.

12. Ng SM, Jin X, Yates R, Kelsall AW. Outcome of noncardiac surgery in children with congenital heart disease performed outside a cardiac center. J Pediatr Surg 2016; 51: 252-6.

13. White MC, Peyton JM. Anaesthetic management of children with congenital heart disease for non-cardiac surgery. Contin Educ Anaesth Crit Care Pain 2012; 12: 17-22.

14. Faraoni D, Vo D, Nasr VG, DiNardo JA. Development and validation of a risk stratification score for children with congenital heart disease undergoing noncardiac surgery. Anesth Analg 2016; 123: 824-30.

15. Torres A Jr, DiLiberti J, Pearl RH, Wohrley J, Raff GW, Bysani GK, et al. Noncardiac surgery in children with hypoplastic left heart syndrome. J Pediatr Surg 2002; 37: 1399-403.

16. Gottlieb EA, Andropoulos DB. Anesthesia for the patient with congenital heart disease presenting for noncardiac surgery. Curr Opin Anaesthesiol 2013; 26: 318-26.

17. Mott AR, Alomrani A, Tortoriello TA, Perles Z, East DL, Stayer SA. Changes in cerebral saturation profile in response to me- 
chanical ventilation alterations in infants with bidirectional superior cavopulmonary connection. Pediatr Crit Care Med 2006; 7: 346-50.

18. Penny DJ, Hayek Z, Redington AN. The effects of positive and negative extrathoracic pressure ventilation on pulmonary blood flow after the total cavopulmonary shunt procedure. Int J Cardiol 1991; 30: 128-30.

19. Redington AN, Penny D, Shinebourne EA. Pulmonary blood flow after total cavopulmonary shunt. Br Heart J 1991; 65: 213 7.

20. Yuki K, Casta A, Uezono S. Anesthetic management of noncardiac surgery for patients with single ventricle physiology. J Anesth 2011; 25: 247-56.

21. Odegard KC, McGowan FX Jr, Zurakowski D, Dinardo JA, Castro RA, del Nido PJ, et al. Procoagulant and anticoagulant factor abnormalities following the Fontan procedure: increased factor VIII may predispose to thrombosis. J Thorac Cardiovasc Surg 2003; 125: 1260-7.

22. Windsor J, Townsley MM, Briston D, Villablanca PA, Alegria JR, Ramakrishna H. Fontan palliation for single-ventricle physiology: perioperative management for noncardiac surgery and analysis of outcomes. J Cardiothorac Vasc Anesth 2017; 31: 2296-303.

23. Chau DF, Gangadharan M, Hartke LP, Twite MD. The post-anesthetic care of pediatric patients with pulmonary hypertension. Semin Cardiothorac Vasc Anesth 2016; 20: 63-73.

24. Abman SH, Hansmann G, Archer SL, Ivy DD, Adatia I, Chung WK, et al. American Heart Association Council on Cardiopul- monary, Critical Care, Perioperative and Resuscitation; Council on Clinical Cardiology; Council on Cardiovascular Disease in the Young; Council on Cardiovascular Radiology and Intervention; Council on Cardiovascular Surgery and Anesthesia; and the American Thoracic Society. Pediatric pulmonary hypertension: guidelines from the American Heart Association and American Thoracic Society. Circulation 2015; 132: $2037-$ 99.

25. Latham GJ, Yung D. Current understanding and perioperative management of pediatric pulmonary hypertension. Paediatr Anaesth 2019; 29: 441-56.

26. Brown ML, DiNardo JA, Nasr VG. Anesthesia in pediatric patients with congenital heart disease undergoing noncardiac surgery: defining the risk. J Cardiothorac Vasc Anesth 2020; 34: 470-8.

27. Matisoff AJ, Olivieri L, Schwartz JM, Deutsch N. Risk assessment and anesthetic management of patients with Williams syndrome: a comprehensive review. Paediatr Anaesth 2015; 25: 1207-15.

28. Kipps AK, Ramamoorthy C, Rosenthal DN, Williams GD. Children with cardiomyopathy: complications after noncardiac procedures with general anesthesia. Paediatr Anaesth 2007; 17: 775-81.

29. Murphy TW, Smith JH, Ranger MR, Haynes SR. General anesthesia for children with severe heart failure. Pediatr Cardiol 2011; 32: 139-44.

30. Ing RJ, Ames WA, Chambers NA. Paediatric cardiomyopathy and anaesthesia. Br J Anaesth 2012; 108: 4-12. 